\title{
A Note on Jordan, Adamović-Mitrinović, and Cusa Inequalities
}

\section{Zhen-Hang Yang and Yu-Ming Chu}

School of Mathematics and Computation Science, Hunan City University, Yiyang 413000, China

Correspondence should be addressed to Yu-Ming Chu; chuyuming2005@126.com

Received 6 November 2013; Accepted 17 February 2014; Published 31 March 2014

Academic Editor: Soon-Yeong Chung

Copyright (C) 2014 Z.-H. Yang and Y.-M. Chu. This is an open access article distributed under the Creative Commons Attribution License, which permits unrestricted use, distribution, and reproduction in any medium, provided the original work is properly cited.

We improve the Jordan, Adamović-Mitrinović, and Cusa inequalities. As applications, several new Shafer-Fink type inequalities for inverse sine function and bivariate means inequalities are established, and a new estimate for sine integral is given.

\section{Introduction}

The classical Jordan inequality [1] is given by

$$
\frac{2}{\pi}<\frac{\sin x}{x}<1
$$

for $x \in(0, \pi / 2)$

Some new developments on refinements, generalizations, and applications for the Jordan inequality can be found in [2] and the references therein.

In the recent past, the following two-side inequality

$$
(\cos x)^{1 / 3}<\frac{\sin x}{x}<\frac{2+\cos x}{3} \quad\left(0<x<\frac{\pi}{2}\right)
$$

has attracted the attention of many researchers (see, e.g., [214]). The left inequality in (2) was obtained by Mitrinović (see [1, p. 238]), while the right one is due to Huygens (see, e.g., [15]) and it is called Cusa inequality [3, 5, 6, 8, 14].

In [16], the following open problem was proposed: for each $p>0$, there are greatest value $q=q(p)$ and least value $r=r(p)$ such that the double inequality

$$
\frac{q \sin x}{1+p \cos x}<x<\frac{r \sin x}{1+p \cos x}
$$

holds for all $x \in(0, \pi / 2)$. This was answered by Carver in [17]. In $[1$, p. $238,3.4 .15]$, it was listed that

$$
\frac{(1+p) \sin x}{1+p \cos x}<x<\frac{(\pi / 2) \sin x}{1+p \cos x}
$$

for $p \in(0,1 / 2]$ and $x \in[0, \pi / 2]$. Wu [18] proved that the double inequality

$$
\frac{(1+p) \cos x}{1+p \cos x}<\frac{\sin x}{x}<\frac{1+q}{1+q \cos x}
$$

holds for $x \in(0, \pi / 2), p \in[-1,2]$, and $q \in[-1 / 4, \infty)$. In particular, he obtained that for $x \in(0, \pi / 2)$,

$$
\frac{3 \cos x}{1+2 \cos x}<\frac{\sin x}{x}<\frac{3}{4-\cos x}
$$

The first inequality in (6) is equivalent to the Huygen inequality:

$$
2 \frac{\sin x}{x}+\frac{\tan x}{x}>3
$$

Jiang [19] showed that for $x \in(0, \pi / 2)$,

$$
\frac{\sin x}{x}>\frac{1+2 \cos x}{2+\cos x} .
$$

$\mathrm{Li}$ and $\mathrm{He}[20]$ gave an improvement of (6) as follows:

$$
\frac{7+5 \cos x}{11+\cos x}<\frac{\sin x}{x}<\frac{9+6 \cos x}{14+\cos x} .
$$


The main purpose of this paper is to give sharp bounds for $(\sin x) / x$ in terms of the functions $H_{1}(\cos t, p)$ and $\mathrm{H}_{2}(\cos t, p)$, where

$$
\begin{aligned}
& H_{1}(x, p)=\frac{2 p+(p+3) x}{3 p+1+2 x}, \quad x \in(0,1), \\
& p \in(-\infty,-1] \cup[0, \infty), \\
& H_{2}(x, p)=\frac{3 p+1}{\pi p} \frac{2 p+(p+3) x}{(3 p+1)+2 x}, \quad x \in(0,1), \\
& p \in(-\infty,-1] \cup(0, \infty) .
\end{aligned}
$$

The rest of this paper is organized as follows. Several lemmas are given in Section 2. Main results and their proofs are given in Section 3, in which Theorem 7 unifies and generalizes Jordan and Cusa inequalities; Theorem 13 shows that Adamović-Mitrinović and Cusa inequalities (2) can be interpolated by $H_{1}(\cos x, p)$ for suitable $p$; Theorem 18 gives a hyperbolic version of Theorem 7. In Section 4, some new Shafer-Fink type inequalities for inverse sine function and several inequalities for bivariate means are presented, and a simpler but more accurate estimate for sine integral is provided.

\section{Lemmas}

Lemma 1. Let $H_{1}$ and $H_{2}$ be defined by (10) and (11), respectively. Then $\mathrm{H}_{1}$ and $\mathrm{H}_{2}$ are, respectively, increasing and decreasing with respect to $p$ on $(-\infty,-1] \cup(0, \infty)$ with the limits

$$
\begin{aligned}
& \lim _{p \rightarrow-\infty} H_{1}(x, p)=\lim _{p \rightarrow \infty} H_{1}(x, p)=\frac{2+x}{3}, \\
& \lim _{p \rightarrow-\infty} H_{2}(x, p)=\lim _{p \rightarrow \infty} H_{2}(x, p)=\frac{2+x}{\pi} .
\end{aligned}
$$

Proof. From (10) and (11) we have

$$
\begin{gathered}
\frac{\partial H_{1}}{\partial p}=\frac{2(x-1)^{2}}{(3 p+1+2 x)^{2}}>0, \\
\frac{\partial H_{2}}{\partial p}=-\frac{3 x}{\pi p^{2}(3 p+2 x+1)^{2}}(p+1) \\
\times((5-2 x) p+(2 x+1)) .
\end{gathered}
$$

If $p \in(0, \infty)$, then we clearly see that $\partial H_{2} / \partial p<0$. If $\in(-\infty,-1)$, then $(5-2 x) p+(2 x+1)<4(x-1)<0$, and then $\partial H_{2} / \partial p<0$.

Simple computations give (12).

Lemma 2. Let $u_{1}, u_{2}$ be defined on $(0,1) \times(-\infty,-1] \cup[0, \infty)$ by

$$
\begin{aligned}
& u_{1}(x, p)=(2 p+(3+p) x)(3 p+1+2 x) \\
& u_{2}(x, p)=2(p+3) x^{3}+8 p x^{2}+2 p(3 p+1) x+3(p+1)^{2}
\end{aligned}
$$

respectively. Then $u_{1}(x, p), u_{2}(x, p)>0$.
Proof. It is not difficult to see that $u_{1}(x, p), u_{2}(x, p)>0$ for $p \in[0, \infty)$. If $p \in(-\infty,-1]$, then

$$
\begin{gathered}
2 p+(3+p) x<2(x-1)<0, \\
(3 p+1+2 x)<2(x-1)<0,
\end{gathered}
$$

and then $u_{1}(x, p)>0$. It remains to prove that $u_{2}(x, p)>0$ for $p \in(-\infty,-1]$. Differentiation leads to

$$
\frac{\partial u_{2}}{\partial p}=(12 x+6) p+\left(2 x^{3}+8 x^{2}+2 x+6\right) \text {. }
$$

Hence, $\partial u_{2} / \partial p<-(12 x+6)+\left(2 x^{3}+8 x^{2}+2 x+6\right)=2 x(x+$ $5)(x-1)<0$, which implies that $u_{2}$ is decreasing in $p$ on $(-\infty,-1)$, and therefore,

$$
u_{2}(x, p)>u_{2}(x,-1)=4 x(x-1)^{2}>0 .
$$

This completes the proof.

Lemma 3. Let $u_{3}$ be defined on $(0,1) \times(-\infty,-1] \cup[0, \infty)$ by

$$
\begin{aligned}
u_{3}(x, p)= & (p+3)^{2} x^{2}+(p+3)(7 p+3) x \\
& +\left(-3 p^{3}+13 p^{2}+21 p+9\right) .
\end{aligned}
$$

Then

(i) $u_{3}(x, p) \geq 0$ for all $x \in(0,1)$ if and only if $p \in$ $\left(-\infty, p_{3}\right]$, where $p_{3} \approx 5.6630$ is the unique solution of the equation $u_{3}(0, p)=-3 p^{3}+13 p^{2}+21 p+9=0$;

(ii) $u_{3}(x, p) \leq 0$ if and only if $p \in[9, \infty)$;

(iii) for every $p \in\left(p_{3}, 9\right)$, there exists a unique $x_{1} \in(0,1)$ such that $u_{3}(x, p)<0$ for $x \in\left(0, x_{1}\right)$ and $u_{3}(x, p)>0$ for $x \in\left(x_{1}, 1\right)$.

Proof. In order to prove the desired results, we need to rewrite $u_{3}(x, p)$ as

$$
u_{3}(x, p)=\left((p+3) x+\frac{7 p+3}{2}\right)^{2}-3\left(p-\frac{9}{4}\right)(p+1)^{2} \text {. }
$$

We clearly see that

$$
\begin{aligned}
& u_{3}(0, p)=-3 p^{3}+13 p^{2}+21 p+9 \\
& u_{3}(1, p)=-3(p+1)^{2}(p-9)
\end{aligned}
$$

We claim that there exists unique $p_{3} \in(5,6)$ such that $u_{3}(0, p)>0$ for $p \in\left(-\infty, p_{3}\right)$ and $u_{3}(0, p)<0$ for $p \in$ $\left(p_{3}, \infty\right)$. Indeed, we have

$$
\begin{aligned}
u_{3}^{\prime}(0, p) & =-9 p^{2}+26 p+21 \\
& =-9\left(p-\frac{13-\sqrt{358}}{9}\right)\left(p-\frac{13+\sqrt{358}}{9}\right),
\end{aligned}
$$


which implies that $u_{3}(0, p)$ is increasing on $((13-\sqrt{358}) / 9$, $(13+\sqrt{358}) / 9)$ and decreasing on $(-\infty,(13-\sqrt{358}) / 9) \cup((13+$ $\sqrt{358}) / 9, \infty)$. Since

$$
\begin{aligned}
u_{3}\left(0, \frac{13-\sqrt{358}}{9}\right) & =\frac{13952}{243}-\frac{716}{243} \sqrt{358} \approx 1.6652>0, \\
u_{3}\left(0, \frac{13+\sqrt{358}}{9}\right) & =\frac{716}{243} \sqrt{358}+\frac{13952}{243}>0, \\
u_{3}(0, \infty) & =-\infty,
\end{aligned}
$$

there exists unique $p_{3} \in((13+\sqrt{358}) / 9, \infty)$ such that $u_{3}\left(0, p_{3}\right)=0$ and $u_{3}(0, p)>0$ for $p \in\left(-\infty, p_{3}\right)$ and $u_{3}(0, p)<0$ for $p \in\left(p_{3}, \infty\right)$. An easy calculation reveals that $p_{3} \approx 5.6630$.

(i) Now we prove the necessary and sufficient condition for $u_{3}(x, p) \geq 0$ for all $x \in(0,1)$. Since $u_{3}(x,-3)=144>$ 0 , we assume that $p \neq-3$. Denote the minimum point of $u_{3}(x, p)$ by $x_{0}$. Then $x_{0}=-(7 p+3) /(2(p+3))$. And then, due to $\partial^{2} u_{3} / \partial x^{2}>0, u_{3}(x, p) \geq 0$ for all $x \in(0,1)$ if and only if at least one of the following cases occur.

Case 1. Consider that $x_{0}=-(7 p+3) /(2(p+3)) \geq 1, u_{3}(1, p) \geq$ 0 . It is derived that $p \in(-3,-1]$.

Case 2. Consider that $x_{0}=-(7 p+3) /(2(p+3)) \leq 0, u_{3}(0, p) \geq$ 0 . It implies that $p \in(-\infty,-3), \cup\left[-3 / 7, p_{3}\right]$.

Case 3. Consider that $x_{0}=-(7 p+3) /(2(p+3)) \in(0,1)$, $u_{3}\left(x_{0}, p\right)=-3(p-9 / 4)(p+1)^{2} \geq 0$. It yields $p \in(-1,-3 / 7)$.

To sum up, $u_{3}(x, p) \geq 0$ for all $x \in(0,1)$ if and only if $p \in\left(-\infty, p_{3}\right]$.

(ii) It is clear that $u_{3}(x, p) \leq 0$ if and only if $u_{3}(0, p) \leq 0$ and $u_{3}(1, p) \leq 0$. Solving the inequalities for $p$ leads to $p \geq 9$.

(iii) In the case of $p \in\left(p_{3}, 9\right)$, we clearly see that $u_{3}(0, p)<$ $0, u_{3}(1, p)>0$, and $x_{0}=-(7 p+3) /(2(p+3))<0$. This implies that there exists a unique $x_{1} \in(0,1)$ such that $u_{3}(x, p)<0$ for $x \in\left(0, x_{1}\right)$ and $u_{3}(x, p)>0$ for $x \in\left(x_{1}, 1\right)$.

This completes the proof.

Now let us consider the sign of function $g$ defined on $(0, \pi / 2) \times(-\infty,-1] \cup[0, \infty)$ by

$$
\begin{gathered}
g(t, p)=t-(((2 p+(p+3) \cos t)(3 p+1+2 \cos t)) \\
\times\left(2(p+3) \cos ^{3} t+8 p \cos ^{2} t\right. \\
\left.\left.\quad+2 p(3 p+1) \cos t+3(p+1)^{2}\right)^{-1} \sin t\right) \\
=t-\frac{u_{1}(\cos t, p)}{u_{2}(\cos t, p)} \sin t,
\end{gathered}
$$

where $u_{1}(x, p)$ and $u_{2}(x, p)$ are defined by (14) and (15), respectively. We have the following.
Lemma 4. Let $g$ be defined on $(0, \pi / 2) \times(-\infty,-1] \cup[0, \infty)$ by (24). Then

(i) $g(t, p)<0$ for all $t \in(0, \pi / 2)$ if and only if $p \in$ $(-\infty,-1] \cup[9, \infty)$;

(ii) $g(t, p)>0$ for all $t \in(0, \pi / 2)$ if and only if $p \in\left[0, p_{1}\right]$, where

$$
p_{1}=\frac{2 \sqrt{6 \pi+1}+3 \pi-2}{12-3 \pi} \approx 6.3433
$$

(iii) in the case of $p \in\left(p_{1}, 9\right)$, there exists a unique $t_{0} \in$ $(0, \pi / 2)$ such that $g(t, p)>0$ for $t \in\left(0, t_{0}\right)$ and $g(t, p)<0$ for $t \in\left(t_{0}, \pi / 2\right)$.

Proof. We first give two limit relations as follows:

$$
\begin{aligned}
\lim _{t \rightarrow 0^{+}} \frac{g(t, p)}{t^{5}} & =-\frac{1}{45} \frac{p-9}{p+1} \quad \text { if } p \neq-1, \\
g\left(\frac{\pi^{-}}{2}, p\right) & =-\frac{12-3 \pi}{6(p+1)^{2}}\left(p-p_{1}\right)\left(p-p_{2}\right) \quad \text { if } p \neq-1,
\end{aligned}
$$

where

$$
\begin{aligned}
& p_{1}=\frac{2 \sqrt{6 \pi+1}+3 \pi-2}{12-3 \pi} \approx 6.3433 \\
& p_{2}=-\frac{2 \sqrt{6 \pi+1}-3 \pi+2}{12-3 \pi}<0
\end{aligned}
$$

In fact, if $p \neq-1$, then making use of power series we get

$$
g(t, p)=-\frac{1}{45} \frac{p-9}{p+1} t^{5}+o\left(t^{5}\right),
$$

which implies the first relation. Direct computations yield the second one.

Differentiating $g(t, p)$ with respect to $t$ leads to

$$
\begin{aligned}
\frac{\partial g}{\partial t} & =1-\frac{u_{1}(\cos t, p)}{u_{2}(\cos t, p)} \cos t+\left.\left(\sin ^{2} t\right) \frac{d}{d x} \frac{u_{1}(x, p)}{u_{2}(x, p)}\right|_{x=\cos t} \\
& =\frac{4(1-x)\left(1-x^{2}\right)}{u_{2}^{2}(x, p)} \times h(x, p),
\end{aligned}
$$

where $u_{1}(x, p)$ and $u_{2}(x, p)$ are defined by (14) and (15), respectively, and

$$
h(x, p)=\left(x+\frac{3 p+1}{2}\right) \times u_{3}(x, p) ;
$$

here $u_{3}(x, p)$ is defined by (19) and $x=\cos t \in(0,1)$.

(i) We now prove that $g(t, p) \leq 0$ for all $t \in(0, \pi / 2)$ if and only if $p \in(-\infty,-1] \cup[9, \infty)$. The necessity easily follows from the inequalities $\lim _{t \rightarrow 0^{+}} t^{-5} g(t, p) \leq 0$ and $g\left(\pi / 2^{-}, p\right) \leq$ 0 if $p \neq-1$ and $g(t,-1)=t-\tan t<0$ together with the relation (26). 
Next we prove the sufficiency. If $p \in[9, \infty)$, then by Lemma $3 u_{3}(x, p) \leq 0$, and then $h(x, p) \leq 0$. This indicates that $g$ is decreasing in $t$ on $(0, \pi / 2)$, and therefore, we get $g(t, p)<g\left(0^{+}, p\right)=0$. If $p \in(-\infty,-1]$, then $u_{3}(x, p) \geq 0$ and $x+(3 p+1) / 2<x-1<0$, which yields $h(x, p) \leq 0$. This also yields that $g$ is decreasing in $t$ on $(0, \pi / 2)$, and so $g(t, p)<g\left(0^{+}, p\right)=0$.

(ii) Similarly, we can prove that $g(t, p)>0$ for all $t \epsilon$ $(0, \pi / 2)$ if and only if $p \in\left[0, p_{1}\right]$. If $g(t, p)>0$ for all $t \in(0, \pi / 2)$, then we have $\lim _{t \rightarrow 0^{+}} t^{-5} g(t, p) \geq 0$ and $g\left(\pi / 2^{-}, p\right) \geq 0$, which together with $(26)$ and $p \in(-\infty,-1] \cup$ $[0, \infty)$ lead to $p \in\left[0, p_{1}\right]$.

In order to prove the sufficiency, we distinguish two cases.

In the case of $p \in\left[0, p_{3}\right]$, by Lemma 3 we have $u_{3}(x, p) \geq$ 0 , which implies that $g$ is increasing in $t$ on $(0, \pi / 2)$, and so, $g(t, p)>g\left(0^{+}, p\right)=0$.

In the case of $p \in\left(p_{3}, p_{1}\right]$, from Lemma 3 there is a unique $x_{1} \in(0,1)$ such that $u_{3}(x, p)<0$ for $x \in\left(0, x_{1}\right)$ and $u_{3}(x, p)>0$ for $x \in\left(x_{1}, 1\right)$. This in conjunction with (30) and (29) shows that $g$ is decreasing in $t$ on $\left(\arccos x_{1}, \pi / 2\right)$ and increasing on $\left(0, \arccos x_{1}\right)$, and consequently, we have

$$
\begin{gathered}
g(t, p)>g\left(0^{+}, p\right)=\text { for } t \in\left(0, \arccos x_{1}\right) \\
g(t, p)>g\left(\frac{\pi^{+}}{2}, p\right)=-\frac{12-3 \pi}{6(p+1)^{2}}\left(p-p_{1}\right)\left(p-p_{2}\right) \geq 0 \\
\text { for } t \in\left(\arccos x_{1}, \frac{\pi}{2}\right),
\end{gathered}
$$

which proves the sufficiency.

(iii) In the case when $p \in\left(p_{1}, 9\right)$, we have seen that $g$ is decreasing in $t$ on $\left(\arccos x_{1}, \pi / 2\right)$ and increasing on $\left(0, \arccos x_{1}\right)$ and $g(t, p)>0$ for $t \in\left(0, \arccos x_{1}\right)$, but

$$
g\left(\frac{\pi^{-}}{2}, p\right)=-\frac{12-3 \pi}{6(p+1)^{2}}\left(p-p_{1}\right)\left(p-p_{2}\right)<0 .
$$

Thus, there is a unique $t_{0} \in\left(\arccos x_{1}, \pi / 2\right)$ such that $g(t, p)>$ 0 for $t \in\left(0, t_{0}\right)$ and $g(t, p)<0$ for $t \in\left(t_{0}, \pi / 2\right)$.

The whole proof is complete.

We next observe the function $f$ defined on $(0, \pi / 2) \times$ $(-\infty,-1] \cup[0, \infty)$ by

$$
\begin{aligned}
f(t, p) & =\ln \frac{\sin t}{t}-\ln H_{1}(\cos t, p) \\
& =\ln \frac{\sin t}{t}-\ln \frac{2 p+(p+3) \cos t}{(3 p+1)+2 \cos t} .
\end{aligned}
$$

Differentiation yields that

$$
\frac{\partial f}{\partial t}=\frac{\cos t}{\sin t}-\frac{1}{t}-\frac{2 \sin t}{3 p+1+2 \cos t}+\frac{(p+3) \sin t}{2 p+(p+3) \cos t}
$$

$$
\begin{aligned}
=( & \left(2(p+3) \cos ^{3} t+\left(3 p^{2}+14 p+3\right) \cos ^{2} t\right. \\
& \left.+3(p+1)^{2}\left(\sin ^{2} t\right)+2 p(3 p+1) \cos t\right) \\
& \left.\times((\sin t)(2 p+3 \cos t+p \cos t)(3 p+2 \cos t+1))^{-1}\right)-\frac{1}{t} \\
= & \frac{1}{\sin t} \frac{u_{2}(\cos t, p)}{u_{1}(\cos t, p)}-\frac{1}{t}=\frac{1}{t \sin t} \frac{u_{2}(\cos t, p)}{u_{1}(\cos t, p)} \times g(t, p),
\end{aligned}
$$

where $u_{1}(x, p), u_{2}(x, p)$, and $g(t, p)$ are defined by (14), (15), and (24), respectively. From Lemmas 2 and 4 the following assertion is immediate.

Lemma 5. Let $f$ be the function defined on $(0, \pi / 2) \times$ $(-\infty,-1] \cup[0, \infty)$ by $(33)$. Then

(i) $f$ is decreasing in $t$ on $(0, \pi / 2)$ if and only if $p \in$ $(-\infty,-1] \cup[9, \infty)$;

(ii) $f$ is increasing in ton $(0, \pi / 2)$ if and only if $p \in\left[0, p_{1}\right]$, where $p_{1} \approx 6.3433$ is given by (25);

(iii) in the case when $p \in\left(p_{1}, 9\right)$, there is a unique $t_{0} \in$ $(0, \pi / 2)$ such that $f$ is increasing in $t$ on $\left(0, t_{0}\right)$ and decreasing on $\left(t_{0}, \pi / 2\right)$.

Lastly, for later use, we also give the following.

Lemma 6. Let $H_{1}$ be defined on $(0,1) \times(-\infty,-1] \cup[0, \infty)$ by (10). Then $H_{1}\left(x^{3}, p\right) \geq x$ if and only if $p \in(-\infty,-1] \cup[1, \infty)$, and $H_{1}\left(x^{3}, p\right) \leq x$ if and only if $p=0$.

Proof. For $p \in(-\infty, \infty)$, we define

$$
\begin{aligned}
u_{4}(x, p) & =2 x^{2}+(1-p) x-2 p \\
& =2\left(x-\frac{p-1}{4}\right)^{2}-\frac{1}{7}\left(14 p+p^{2}+1\right) .
\end{aligned}
$$

Then $u_{4}(x, p) \geq 0$ holds for all $x \in(0,1)$ if and only if $p \in$ $(-\infty, 0]$.

In fact, $u_{4}(x, p) \geq 0$ if and only if at least one case of the following occurs.

Case 1. Consider that $(p-1) / 4 \geq 1, u_{4}(1, p)=3-3 p \geq 0$. It is impossible.

Case 2. Consider that $(p-1) / 4 \leq 0, u_{4}(0, p)=-2 p \geq 0$. It indicates $p \in(-\infty, 0]$.

Case 3. Consider that $0<(p-1) / 4<1, u_{4}((p-1) / 4, p) \geq 0$. It is impossible.

In the same way, we can prove that $u_{4}(x, p) \leq 0$ holds for all $x \in(0,1)$ if and only if $p \in[1, \infty)$. 
We now prove that $H_{1}\left(x^{3}, p\right) \geq x$ if and only if $p \in$ $(-\infty,-1] \cup[1, \infty)$. Factoring yields

$$
\begin{aligned}
H_{1}\left(x^{3}, p\right)-x & =-2(x-1)^{2} \frac{2 x^{2}+(1-p) x-2 p}{3 p+2 x^{3}+1} \\
& =-(x-1)^{2} \frac{u_{4}(x, p)}{3 p+2 x^{3}+1} .
\end{aligned}
$$

If $p \in(-\infty,-1]$, then $3 p+2 x^{3}+1<0$, and then, $H_{1}\left(x^{3}, p\right) \geq x$ if and only if $u_{4}(x, p) \geq 0$, which is equivalent to $p \in(-\infty,-1] \cap(-\infty, 0]=(-\infty,-1]$. If $p \in[0, \infty)$, then $3 p+2 x^{3}+1>0$, and then, $H_{1}\left(x^{3}, p\right) \geq x$ if and only if $u_{4}(x, p) \leq 0$, which is equivalent to $p \in[0, \infty) \cap$ $[1, \infty)=[1, \infty)$. Consequently, $H_{1}\left(x^{3}, p\right) \geq x$ if and only if $p \in(-\infty,-1] \cup[1, \infty)$.

Next we show that $H_{1}\left(x^{3}, p\right) \leq x$ if and only if $p=0$. In fact, if $p \in(-\infty,-1]$, then $H_{1}\left(x^{3}, p\right) \leq x$ if and only if $u_{4}(x, p) \leq 0$, which yields $p \in[1, \infty)$. It is clearly a contradiction. If $p \in[0, \infty)$, then the statement in question if and only if $u_{4}(x, p) \geq 0$, which leads to $p \in[0, \infty) \cap(-\infty, 0]=$ $\{0\}$. Thus the proof is complete.

\section{Main Results}

Theorem 7. Let $p \in(-\infty,-1] \cup[0, \infty)$. Then for $t \in(0, \pi / 2)$,

$$
\frac{\sin t}{t}<\frac{2 p+(p+3) \cos t}{(3 p+1)+2 \cos t}
$$

holds if and only if $p \in(-\infty,-1] \cup[9, \infty)$. Moreover, we have

$$
\begin{aligned}
H_{2}(\cos t, p) & =\lambda_{p} \frac{2 p+(p+3) \cos t}{(3 p+1)+2 \cos t}<\frac{\sin t}{t} \\
& <\frac{2 p+(p+3) \cos t}{(3 p+1)+2 \cos t}=H_{1}(\cos t, p)
\end{aligned}
$$

for $p \in(-\infty,-1] \cup[9, \infty)$, where $\lambda_{p}=(3 p+1) /(\pi p)$ is the best possible. And the lower and upper bounds in (38) are decreasing and increasing in $p$ on $(-\infty,-1] \cup(0, \infty)$, respectively.

Proof. Clearly, the desired result is equivalent to $f(t, p)<0$ if and only if $p \in(-\infty,-1] \cup[9, \infty)$, where $f(t, p)$ is defined by (33). To this end, we give two limit relations. The first one follows by expanding $f(t, p)$ in power series for $t$. We have

$$
f(t, p)=-\frac{1}{180} \frac{p-9}{p+1} t^{4}+o\left(t^{4}\right) \quad \text { if } p \neq-1
$$

which yields

$$
\lim _{t \rightarrow 0^{+}} \frac{f(t, p)}{t^{4}}=-\frac{1}{180} \frac{p-9}{p+1} \text { if } p \neq-1 .
$$

The second one is derived by a simple computation; that is,

$$
f\left(\frac{\pi^{-}}{2}, p\right)=\ln \frac{3 p+1}{\pi p} .
$$

Now we prove that $f(t, p)<0$ for all $t \in(0, \pi / 2)$ if and only if $p \in(-\infty,-1] \cup[9, \infty)$.

The necessity easily follows by solving the simultaneous inequalities:

$$
\begin{aligned}
\lim _{t \rightarrow 0^{+}} \frac{f(t, p)}{t^{4}} & =-\frac{1}{180} \frac{p-9}{p+1} \leq \text { if } p \neq-1, \\
f(t,-1) & =\ln \frac{\sin t}{t}<0, \\
f\left(\frac{\pi^{-}}{2}, p\right) & =\ln \frac{3 p+1}{\pi p} \leq 0,
\end{aligned}
$$

which implies $p \in(-\infty,-1] \cup[9, \infty)$.

The sufficiency is due to Lemma 5. In fact, If $p \in$ $(-\infty,-1] \cup[9, \infty)$, then by Lemma 5 we see that $f$ is decreasing in $t$ on $(0, \pi / 2)$. Hence, $f(t, p)<f\left(0^{+}, p\right)=0$.

Utilizing the monotonicity of $f$ in $t$ on $(0, \pi / 2)$ gives (38). And from Lemma 1 it is seen that the lower and upper bounds in (38) are decreasing and increasing in $p$ on $(-\infty,-1] \cup$ $(0, \infty)$, respectively.

Thus the proof is finished.

By Theorem 7 and Lemma 1, we have the following interesting chain of inequalities.

Corollary 8. For $t \in(0, \pi / 2)$, one has

$$
\begin{aligned}
\frac{2}{\pi} & =H_{2}(\cos t,-1)<\cdots<H_{2}(\cos t,-\infty) \\
& =\frac{2+\cos t}{\pi}=H_{2}(\cos t, \infty)<\cdots<H_{2}(\cos t, 9)<\frac{\sin t}{t} \\
& <H_{1}(\cos t, 9)<\cdots<H_{1}(\cos t, \infty)=\frac{2+\cos t}{3} \\
& =H_{1}(\cos t,-\infty) \cdots<H_{1}(\cos t,-1)=-1 .
\end{aligned}
$$

Remark 9. It is clear that our results unify and refine Jordan and Cusa's inequalities and show that the first one in (9) is sharp. Also, Theorem 7 contains other known results, for example, taking $p=-3$ in (38) we get

$$
\frac{8}{\pi} \frac{1}{4-\cos t}<\frac{\sin t}{t}<3 \frac{1}{4-\cos t},
$$

which contain (6). After a simple transformation, (44) can be written as

$$
\frac{8}{\pi} \frac{t}{\sin t}+\cos t<4<3 \frac{t}{\sin t}+\cos t
$$

where the second inequality in (45) is due to Neuman and Sándor $[6,(2.12)]$.

Theorem 10. Let $p \in(-\infty,-1] \cup[0, \infty)$. Then for $t \in(0, \pi / 2)$

$$
\frac{2 p+(p+3) \cos t}{(3 p+1)+2 \cos t}<\frac{\sin t}{t}
$$

holds if and only if $p \in\left[0, p_{0}\right]$, where $p_{0}=(\pi-3)^{-1} \approx 7.0625$. 
Moreover, for $p \in\left(0, p_{1}\right]$, one has

$$
\begin{aligned}
H_{1}(\cos t, p) & =\frac{2 p+(p+3) \cos t}{(3 p+1)+2 \cos t}<\frac{\sin t}{t} \\
& <\lambda_{p} \frac{2 p+(p+3) \cos t}{(3 p+1)+2 \cos t}=H_{2}(\cos t, p),
\end{aligned}
$$

where $p_{1} \approx 6.3433, \lambda_{p}=(3 p+1) /(\pi p)$ is the best possible. And $H_{1}(\cos t, p), H_{2}(\cos t, p)$ are decreasing and increasing in $p$ on $(-\infty,-1] \cup(0, \infty)$, respectively.

For $p \in\left(p_{1}, p_{0}\right]$ one has

$$
\begin{aligned}
H_{1}(\cos t, p) & =\frac{2 p+(p+3) \cos t}{(3 p+1)+2 \cos t}<\frac{\sin t}{t} \\
& <\delta_{p} \frac{2 p+(p+3) \cos t}{(3 p+1)+2 \cos t}=\delta_{p} H_{1}(\cos t, p),
\end{aligned}
$$

where $\delta_{p}=\left(\sin t_{0} / t_{0}\right)\left(\left((3 p+1)+2 \cos t_{0}\right) /\left(2 p+(p+3) \cos t_{0}\right)\right)$ is the best possible and $t_{0}$ is the unique root of the equation

$$
\begin{aligned}
& \frac{(2 p+(3+p) \cos t)(3 p+1+2 \cos t)}{2(p+3) \cos ^{3} t+8 p \cos ^{2} t+2 p(3 p+1) \cos t+3(p+1)^{2}} \\
& \times \sin t=t
\end{aligned}
$$

on $(0, \pi / 2)$.

Proof. Since the inequality (46) is equivalent to $f(t, p)>0$, it suffices to prove that $f(t, p)>0$ holds for $t \in(0, \pi / 2)$ if and only if $p \in\left[0, p_{0}\right]$.

Similarly, solving the simultaneous inequalities $\lim _{t \rightarrow 0} t^{-4} f(t, p) \geq 0$ and $f\left(\pi / 2^{-}, p\right) \geq 0$ with $p \in(-\infty,-1] \cup(0, \infty)$ yields $p \in\left[0, p_{0}\right]$, which proves the necessity.

Conversely, the condition $p \in\left[0, p_{0}\right]$ is also sufficient for $f(t, p)>0$ to be valid. For this end, we divide the proof into two cases.

Case 1. Consider that $p \in\left[0, p_{1}\right]$. By Lemma 5 it is seen that $f$ is increasing in $t$ on $(0, \pi / 2)$, which indicates that $f(t, p)>$ $f\left(0^{+}, p\right)=0$.

Case 2. Consider that $p \in\left(p_{1}, p_{0}\right]$. By Lemma 5 we see that there is a unique $t_{0} \in(0, \pi / 2)$ such that $f$ is increasing in $t$ on $\left(0, t_{0}\right)$ and decreasing on $\left(t_{0}, \pi / 2\right)$. It is acquired that

$$
\begin{array}{r}
f\left(t_{0}, p\right)>f(t, p)>f\left(0^{+}, p\right)=0 \quad \text { for } t \in\left(0, t_{0}\right), \\
f\left(t_{0}, p\right)>f(t, p)>f\left(\pi / 2^{-}, p\right)=\ln \frac{3 p+1}{\pi p} \geq 0 \\
\text { for } t \in\left(t_{0}, \frac{\pi}{2}\right) ;
\end{array}
$$

that is,

$$
f\left(t_{0}, p\right) \geq f(t, p)>\quad \text { for } t \in(0, \pi / 2),
$$

which proves the sufficiency.
In the first case, application of the monotonicity of $f$ in $t$ on $(0, \pi / 2)$ leads to $(47)$, and $\lambda_{p}=(3 p+1) /(\pi p)$. In the second case, (51) also yields (47), and

$$
\delta_{p}=\exp f\left(t_{0}, p\right)=\frac{\sin t_{0}}{t_{0}} \frac{(3 p+1)+2 \cos t_{0}}{2 p+(p+3) \cos t_{0}} .
$$

Thus we complete the proof.

Remark 11. Taking $p=7$ in (46), we get the first inequality in (9).

Letting $p=p_{0}=(\pi-3)^{-1}$ and solving (49) by mathematical computation software, we find that $t_{0} \approx 1.3055$ and $\delta_{p_{0}} \approx 1.0015$. Letting $p=p_{1}$ be defined by (25) yields $\lambda_{p_{1}}=\left(3 p_{1}+1\right) /\left(\pi p_{1}\right) \approx 1.0051$. By Theorem 10 we get the following.

Corollary 12. For $t \in(0, \pi / 2)$, one has

$$
\begin{aligned}
& \frac{2 p_{0}+\left(p_{0}+3\right) \cos t}{\left(3 p_{0}+1\right)+2 \cos t}<\frac{\sin t}{t}<\delta_{p_{0}} \frac{2 p_{0}+\left(p_{0}+3\right) \cos t}{\left(3 p_{0}+1\right)+2 \cos t} \\
& \frac{2 p_{1}+\left(p_{1}+3\right) \cos t}{\left(3 p_{1}+1\right)+2 \cos t}<\frac{\sin t}{t}<\lambda_{p_{1}} \frac{2 p_{1}+\left(p_{1}+3\right) \cos t}{\left(3 p_{1}+1\right)+2 \cos t}
\end{aligned}
$$

where $\delta_{p_{0}} \approx 1.0015$ and $\lambda_{p_{1}} \approx 1.0051$ are the best possible constants.

Letting $x=\cos ^{1 / 3} t$ in Lemma 6 and using Theorems 7 and 10, we obtain a chain of inequalities that interpolates Adamović-Mitrinović and Cusa's inequalities (2) by $H_{1}(\cos x, p)$.

Theorem 13. For $t \in(0, \pi / 2)$, the inequalities

$$
\begin{aligned}
\frac{2 p+(p+3) \cos t}{(3 p+1)+2 \cos t} & <\cos ^{1 / 3} t<\frac{2 q+(q+3) \cos t}{(3 q+1)+2 \cos t} \\
& <\frac{\sin t}{t}<\frac{2 r+(r+3) \cos t}{(3 r+1)+2 \cos t}<\frac{2+\cos t}{3} \\
& <\frac{2 s+(s+3) \cos t}{(3 s+1)+2 \cos t}
\end{aligned}
$$

hold if and only if $p=0, q \in\left[0, p_{0}\right], r \in[9, \infty)$, and $s \in$ $(-\infty,-1]$, where $p_{0}=(\pi-3)^{-1}$.

Using the monotonicity of $f(t, p)$ in $t$ on $(0, \pi / 4)$ given by parts one and two of Lemma 5, we see that

$$
\begin{aligned}
\ln ( & \left.\frac{4}{\pi} \frac{3 p+\sqrt{2}+1}{(2 \sqrt{2}+1) p+3}\right) \\
= & f\left(\frac{\pi}{4}, p\right) \leq f\left(\frac{t}{2}, p\right)=\ln \frac{2 \sin (t / 2)}{t} \\
& -\ln H_{1}\left(\cos \frac{t}{2}, p\right) \leq f(0, p)=0
\end{aligned}
$$


hold for $p \in(-\infty,-1] \cup[9, \infty)$. And then we have

$$
\begin{gathered}
\frac{4}{\pi} \frac{3 p+\sqrt{2}+1}{(2 \sqrt{2}+1) p+3} H_{1}\left(\cos \frac{t}{2}, p\right) \cos \frac{t}{2} \\
<\frac{\sin t}{t}=H_{1}\left(\cos \frac{t}{2}, p\right) \cos \frac{t}{2}
\end{gathered}
$$

It is clear that the right-hand in (56) is increasing in $p$ on $(-\infty,-1] \cup[0, \infty)$, but the monotonicity of left-hand is to be checked. We define

$$
H_{3}(x, p)=\frac{4}{\pi} \frac{3 p+\sqrt{2}+1}{(2 \sqrt{2}+1) p+3} H_{1}(x, p),
$$

where $x=\cos (t / 2) \in[1 / \sqrt{2}, 1]$. Logarithmic differentiation leads to

$$
\begin{aligned}
& \frac{\partial \ln H_{3}}{\partial p} \\
& =\frac{3}{(3 p+\sqrt{2}+1)}-\frac{2 \sqrt{2}+1}{(p(2 \sqrt{2}+1)+3)} \\
& \quad-\frac{3}{(3 p+2 x+1)}+\frac{x+2}{2 p+x(p+3)} \\
& =-\left(\left(6(2 \sqrt{2}+1)\left(x-\frac{\sqrt{2}}{2}\right)\left(\frac{22-9 \sqrt{2}}{7}-x\right)\right)\right. \\
& \quad \times((3 p+\sqrt{2}+1)(p(2 \sqrt{2}+1)+3)(3 p+2 x+1) \\
& \left.\quad \times(2 p+x(p+3)))^{-1}\right)(p+1)\left(p-u_{5}(x)\right),
\end{aligned}
$$

where

$$
u_{5}(x)=\frac{(5-2 \sqrt{2}) x-(\sqrt{2}+2)}{(5 \sqrt{2}-2)-(2 \sqrt{2}+1) x}
$$

Since

$$
u_{5}^{\prime}(x)=-\frac{12(3-2 \sqrt{2})}{(5 \sqrt{2}-2-(2 \sqrt{2}+1) x)^{2}}<0
$$

we have $-1=u_{5}(1)<u_{5}(x)<u_{5}(1 / \sqrt{2})=-(24 \sqrt{2}+5) / 49 \approx$ -0.7947 . Consequently, $\partial\left(\ln H_{3}\right) / \partial p<0$ for $p \in(-\infty,-1] \cup$ $[0, \infty)$.

The result can be stated as a theorem.

Theorem 14. Let $p \in(-\infty,-1] \cup[0, \infty)$. Then for $t \in(0, \pi / 2)$ the inequalities

$$
\begin{aligned}
\sigma_{p} \frac{2 p \cos (t / 2)+(p+3) \cos ^{2}(t / 2)}{(3 p+1)+2 \cos (t / 2)} \\
<\frac{\sin t}{t}<\frac{2 p \cos (t / 2)+(p+3) \cos ^{2}(t / 2)}{(3 p+1)+2 \cos (t / 2)} .
\end{aligned}
$$

hold if and only if $p \in(-\infty,-1] \cup[9, \infty)$, where $\sigma_{p}=$ $(4 / \pi)((3 p+\sqrt{2}+1) /((2 \sqrt{2}+1) p+3))$ is the best constant. And the right-hand and left-hand in (61) are increasing and decreasing in p, respectively. Inequality (61) is reversed if and only if $p \in\left[0, p_{1}\right]$, where $p_{1} \approx 6.3433$ is defined by (25).

Putting $p=9, \infty, 0,1$ in Theorem 14 we have the following.

Corollary 15. For $t \in(0, \pi / 2)$ the following inequalities hold:

$$
\begin{gathered}
\frac{2(41 \sqrt{2}-25)}{7 \pi} \frac{2 \cos ^{2}(t / 2)+3 \cos (t / 2)}{\cos (t / 2)+14} \\
<\frac{\sin t}{t}<3 \frac{2 \cos ^{2}(t / 2)+3 \cos (t / 2)}{\cos (t / 2)+14}, \\
\frac{4(2 \sqrt{2}-1)}{7} \frac{\cos ^{2}(t / 2)+2 \cos (t / 2)}{\pi} \\
<\frac{\sin t}{t}<\frac{\cos ^{2}(t / 2)+2 \cos (t / 2)}{3}, \\
3 \frac{\cos (t / 2)}{2 \cos ^{2}(t / 2)+1}<\frac{\sin t}{t}<\frac{4(\sqrt{2}+1)}{\pi} \frac{\cos ^{2}(t / 2)}{2 \cos ^{2}(t / 2)+1}, \\
\frac{2 \cos ^{2}(t / 2)+1}{\cos ^{2}(t / 2)+2}<\frac{\sin t}{t}<\frac{2(3-\sqrt{2})}{\pi} \frac{2 \cos (t / 2)+1}{\cos ^{2}(t / 2)+2} .
\end{gathered}
$$
by

Further, let $H_{4}$ be defined on $[1 / \sqrt{2}, 1] \times(-\infty,-1] \cup[0, \infty)$

$$
H_{4}(x, p)=\frac{H_{1}\left(2 x^{2}-1, p\right)}{x H_{1}(x, p)},
$$

where $H_{1}$ is defined by (10). We can show that the monotonicity of $H_{4}$ in $x$ for certain fixed $p$. Differentiation again yields

$$
\begin{aligned}
\frac{\partial \ln H_{4}(x, p)}{\partial x}= & \frac{2}{1+3 p+2 x}-\frac{1}{x}-\frac{p+3}{2 p+(p+3) x} \\
& +\frac{4(p+3) x}{(p-3)+2(p+3) x^{2}}-\frac{8 x}{4 x^{2}+3 p-1}
\end{aligned}
$$

It is easy to verify that

$$
\begin{aligned}
& \frac{\partial \ln H_{4}(x, 9)}{\partial x} \\
& =-2 \frac{(x-1)^{2}\left(594 x^{2}+240 x^{3}+8 x^{4}+910 x+273\right)}{x(2 x+3)(x+14)\left(2 x^{2}+13\right)\left(4 x^{2}+1\right)} \\
& <0, \\
& \quad \frac{\partial \ln H_{4}(x, \infty)}{\partial x}=-2 \frac{(1-x)(2 x+1)}{x(x+2)\left(2 x^{2}+1\right)}<0, \\
& \frac{\partial \ln H_{4}(x, 1)}{\partial x}=2 \frac{(1-x)\left(2 x^{3}+8 x^{2}+x+1\right)}{x(2 x-1)(x+2)\left(2 x^{2}+1\right)}>0 .
\end{aligned}
$$


Consequently, we have

$$
\begin{aligned}
1 & =\frac{H_{1}(1, p)}{H_{1}(1, p)}<\frac{H_{1}\left(2 x^{2}-1, p\right)}{x H_{1}(x, p)} \\
< & \frac{H_{1}(0, p)}{(1 / \sqrt{2}) H_{1}(1 / \sqrt{2}, p)}=\frac{4 p}{3 p+1} \frac{3 p+1+\sqrt{2}}{(2 \sqrt{2}+1) p+3} \\
& \text { for } p=9, \infty .
\end{aligned}
$$

It is reversed for $p=1$. From these we can obtain the following.

Theorem 16. For $t \in(0, \pi / 2)$ the following inequalities hold:

$$
\begin{aligned}
\frac{28}{9 \pi} \frac{6 \cos t+9}{\cos t+14} & <\frac{41(2 \sqrt{2}-25)}{7 \pi} \frac{2 \cos ^{2}(t / 2)+3 \cos (t / 2)}{\cos (t / 2)+14} \\
& <\frac{\sin t}{t}<\frac{6 \cos ^{2}(t / 2)+9 \cos (t / 2)}{\cos (t / 2)+14} \\
& <\frac{6 \cos t+9}{\cos t+14}, \\
\frac{2+\cos t}{\pi} & <\frac{12(2 \sqrt{2}-1)}{7 \pi} \frac{\cos ^{2}(t / 2)+2 \cos (t / 2)}{3} \\
& <\frac{\sin t}{t}<\frac{\cos ^{2}(t / 2)+2 \cos (t / 2)}{3} \\
& <\frac{2+\cos t}{3}, \\
\frac{2 \cos t+1}{\cos t+2} & <\frac{2 \cos (t / 2)+1}{\cos (t / 2)+2}<\frac{\sin t}{t} \\
& <\frac{2(3-\sqrt{2})}{\pi} \frac{2 \cos ^{2}(t / 2)+1}{\cos (t / 2)+2} \\
& <\frac{4 \frac{2 \cos t+1}{\cos t+2} .}{2}
\end{aligned}
$$

Additionally, Lemma 4 implies an optimal two-side inequality.

Theorem 17. Let $p \in(-\infty,-1] \cup[0, \infty)$ and let $u_{1}(x, p)$ and $u_{2}(x, p)$ be defined by (14) and (15), respectively. Then for $t \in$ $(0, \pi / 2)$ the two-side inequality

$$
\frac{u_{2}(\cos t, p)}{u_{1}(\cos t, p)}<\frac{\sin t}{t}<\frac{u_{2}(\cos t, q)}{u_{1}(\cos t, q)}
$$

holds if and only if $p \in(-\infty,-1] \cup[9, \infty)$ and $q \in\left[0, p_{1}\right]$, where $p_{1} \approx 6.3433$. And, for $x \in(0,1)$, the function $p \mapsto$ $u_{2}(x, p) / u_{1}(x, p)$ is decreasing on $(-\infty,-1] \cup[0, \infty)$.

Proof. Since $u_{1}(x, p), u_{2}(x, p)>0$ for $p \in(-\infty,-1] \cup[0, \infty)$ and $x \in(0,1)$ by Lemma 2 and $g(t, p)$ defined by $(24)$ can be written as

$$
g(t, p)=-t \frac{u_{1}(\cos t, p)}{u_{2}(\cos t, p)}\left(\frac{\sin t}{t}-\frac{u_{2}(\cos t, p)}{u_{1}(\cos t, p)}\right),
$$

it follows from Lemma 4 that (71) $\mathrm{k}$ holds if and only if $p \in(-\infty,-1] \cup[9, \infty)$ and $q \in\left[0, p_{1}\right]$. It remains to check the monotonicity of $u_{2}(\cos t, p) / u_{1}(\cos t, p)$ in $p$. Differentiation yields

$$
\begin{aligned}
\frac{d}{d p} \frac{u_{2}(x, p)}{u_{1}(x, p)}= & -6(x+1)(x-1)^{2} \\
& \times \frac{(p+1)((5+x) p+5 x+1)}{(2 p+3 x+p x)^{2}(3 p+2 x+1)^{2}},
\end{aligned}
$$

where $x \in(0,1)$. If $p \in[0, \infty)$, then the numerator of the fraction in right-hand above is clearly positive. Consider that $(p+1)((5+x) p+5 x+1)>0$. If $p \in(-\infty,-1]$, then $(p+1) \leq 0$ and $((5+x) p+5 x+1) \leq 5(x-1)<0$, which yields that the numerator is nonnegative.

This proves the assertion.

Similarly, we can obtain a hyperbolic version of Theorems 7 and 10

Theorem 18. Let $p \in(-\infty,-1] \cup[0, \infty)$. Then for $t \in(0, \infty)$

$$
\frac{2+(1+3 p) \cosh t}{3+p+2 p \cosh t}<\frac{\sinh t}{t}
$$

holds if and only if $p \in(-\infty,-1] \cup[1 / 9, \infty)$. It is reversed if and only if $p=0$.

Proof. Let $F$ be the function defined on $(0, \infty) \times(-\infty,-1] \cup$ $[0, \infty)$ by

$$
F(t, p)=\frac{3+p+2 p \cosh t}{2+(1+3 p) \cosh t} \sinh t-t .
$$

Then the inequalities (74) are equivalent to $F(t, p)>0$. Expanding in power series yields

$$
F(t, p)=\frac{t^{5}}{180} \frac{9 p-1}{p+1}+o\left(t^{5}\right)
$$

which implies

$$
\begin{aligned}
\lim _{t \rightarrow 0} \frac{F(t, p)}{t^{5}} & =\frac{1}{20} \frac{p-1 / 9}{p+1} \text { if } p \neq-1, \\
F(t,-1) & =\sinh t-t>0 .
\end{aligned}
$$

On the other hand, we have

$$
\lim _{t \rightarrow \infty} \frac{F(t, p)}{\sinh t}=\frac{2 p}{1+3 p} .
$$

Now we prove desired results.

(i) We first prove that $F(t, p)>0$ holds if and only if $p \in$ $(-\infty,-1] \cup[1 / 9, \infty)$.

If $F(t, p)>0$ for all $t>0$, then we have

$$
\begin{aligned}
\lim _{t \rightarrow 0} \frac{F(t, p)}{t^{5}} & =\frac{1}{20} \frac{p-1 / 9}{p+1} \geq 0, \\
F(t,-1) & =\sinh t-t>0, \\
\lim _{t \rightarrow \infty} \frac{F(t, p)}{\sinh t} & =\frac{2 p}{1+3 p} \geq 0 .
\end{aligned}
$$

Solving the inequalities yields $p \in(-\infty,-1] \cup[1 / 9, \infty)$. 
We prove the condition $p \in(-\infty,-1] \cup[1 / 9, \infty)$ is sufficient for $F(t, p)>0$ to hold for $t \in(0, \infty)$. Differentiation gives

$$
\begin{aligned}
\frac{\partial F}{\partial t}= & \frac{(3 p+1+2 \cosh t)}{2 p+(p+3) \cosh t} \cosh t \\
& -\frac{3(p+1)^{2} \sinh ^{2} t}{(2 p+(p+3) \cosh t)^{2}}-1 \\
= & (x-1)^{2} \frac{2 p(3 p+1) x+\left(3 p^{2}+6 p-1\right)}{(x+3 p x+2)^{2}},
\end{aligned}
$$

where $x=\cosh t \in(1, \infty)$.

Due to $p \in(-\infty,-1] \cup[1 / 9, \infty)$, we see that $2 p(3 p+1)>$ 0 , which yields

$$
\begin{aligned}
2 p & (3 p+1) x+\left(3 p^{2}+6 p-1\right) \\
> & 2 p(3 p+1)+\left(3 p^{2}+6 p-1\right) \\
& =(p+1)(9 p-1) \geq 0 .
\end{aligned}
$$

Then $\partial F / \partial t>0$; that is, $F$ is increasing in $t$ on $(0, \infty)$. It is obtained that $F(t, p)>F(0, p)=0$, which proves the sufficiency.

(ii) Next we prove that the reverse inequality of (74) holds if and only if $p=0$. The necessity follows from

$$
\begin{aligned}
& \lim _{t \rightarrow 0} \frac{F(t, p)}{t^{5}}=\frac{1}{20} \frac{p-1 / 9}{p+1} \leq 0, \\
& \lim _{t \rightarrow \infty} \frac{F(t, p)}{\sinh t}=\frac{2 p}{1+3 p} \leq 0,
\end{aligned}
$$

and the assumption $p \in(-\infty,-1] \cup[0, \infty)$. We get $p=0$.

Now we prove $F(t, p)<0$ when $p=0$. We have

$$
\frac{\partial F}{\partial t}=-\frac{(x-1)^{2}}{(x+3 p x+2)^{2}}<0,
$$

where $x=\cosh t \in(1, \infty)$, then $F(t, 0)<F(0,0)=0$.

Thus the proof of Theorem 18 is complete.

Denote

$$
H_{5}(x, p)=\frac{2+(1+3 p) x}{3+p+2 p x} .
$$

It is easy to verify that $H_{5}(x, p)=H_{1}\left(x, p^{-1}\right)$ for $p \neq 0$. By Lemma 1 , we see that $H_{5}$ is decreasing in $p$ on $(-\infty,-1] \cup$ $[0, \infty)$. Thus, as a consequence of Theorem 14 , we have the following.

\section{Corollary 19. One has}

$$
\begin{aligned}
\frac{2+\cosh t}{3} & >\frac{\sinh t}{t}>H_{5}\left(\cosh t, \frac{1}{9}\right) \\
& >\cdots>H_{5}(\cosh t, \infty)=\frac{3 \cosh t}{2 \cosh t+1} \\
& =H_{5}(\cosh t,-\infty)>\cdots H_{5}(\cosh t,-1)=1 .
\end{aligned}
$$

Furthermore, note that $H_{5}(x, p)=H_{1}^{-1}\left(x^{-1}, p\right)$ and by Lemma 6 we have the following.

Corollary 20. One has

$$
\begin{aligned}
\frac{2+\cosh t}{3} & >\frac{\sinh t}{t}>\cosh ^{1 / 3} t>\frac{1+2 \cosh t}{2+\cosh t} \\
& >H_{5}(\cosh t, p),
\end{aligned}
$$

where $p \in(-\infty,-1] \cup(1, \infty)$.

\section{Applications}

In this section, we give some applications of our results.

4.1. Shafer-Fink Type Inequalities. In [1, p. 247, 3.4.31], it was listed that the inequality

$$
\arcsin x>\frac{6(\sqrt{x+1}-\sqrt{1-x})}{4+\sqrt{x+1}+\sqrt{1-x}}>\frac{3 x}{2+\sqrt{1-x^{2}}}
$$

holds for $x \in(0,1)$, which is due to Shafer [21]. Fink [22] proved that the double inequality

$$
\frac{3 x}{2+\sqrt{1-x^{2}}} \leq \arcsin x \leq \frac{\pi x}{2+\sqrt{1-x^{2}}}
$$

is true for $x \in[0,1]$. There has been some improvements and generalizations of Shafer-Fink inequality (see [23]). Letting $\sin t=x$ in Theorems $7,10,13,14,16$ and 17 we can obtain corresponding Shafer-Fink type inequalities, which clearly contain many known results. For example, Theorems 7 and 10 can be changed into the following.

Proposition 21. For $x \in(0,1)$, the two-side inequality

$$
\begin{aligned}
& \frac{x}{H_{1}\left(\sqrt{1-x^{2}}, p\right)} \\
& =x \frac{(3 p+1)+2 \sqrt{1-x^{2}}}{2 p+(p+3) \sqrt{1-x^{2}}}<\arcsin x \\
& <\frac{\pi p}{3 p+1} x \frac{(3 p+1)+2 \sqrt{1-x^{2}}}{2 p+(p+3) \sqrt{1-x^{2}}} \\
& =\frac{x}{H_{2}\left(\sqrt{1-x^{2}}, p\right)}
\end{aligned}
$$

holds if and only if $p \in(-\infty,-1] \cup[9, \infty)$, where $\pi p /(3 p+1)$ is the best possible. And, the lower and upper bounds in (89) are decreasing and increasing in $p$ on $(-\infty,-1] \cup(0, \infty)$, respectively.

Inequality (89) is reversed if $p \in\left[0, p_{1}\right]$, where $p_{1} \approx 6.3433$ is defined by (25).

Letting $\sin t=x$, then $\cos (t / 2)=(1 / 2)(\sqrt{1+x}+\sqrt{1-x})$. Theorem 14 can be restated as follows. 
Proposition 22. For $x \in(0,1)$, the two-side inequality

$$
\begin{aligned}
& 2 \frac{(3 p+1)(\sqrt{1+x}-\sqrt{1-x})+2 x}{4 p+(p+3)(\sqrt{1+x}+\sqrt{1-x})} \\
& \quad<\arcsin x<\frac{2}{\sigma_{p}} \frac{(3 p+1)(\sqrt{1+x}-\sqrt{1-x})+2 x}{4 p+(p+3)(\sqrt{1+x}+\sqrt{1-x})}
\end{aligned}
$$

holds if and only if $p \in(-\infty,-1] \cup[9, \infty)$, where $\sigma_{p}=$ $(4 / \pi)((3 p+\sqrt{2}+1) /((2 \sqrt{2}+1) p+3))$ is the best constant. And, the lower and upper bounds in (90) are decreasing and increasing in $p$ on $(-\infty,-1] \cup(0, \infty)$, respectively.

Inequality $(90)$ is reversed if $p \in\left[0, p_{1}\right]$, where $p_{1} \approx 6.3433$ is defined by (25).

As another example, Theorem 16 can be rewritten as follows.

Proposition 23. For $x \in(0,1)$, all the following chains of inequalities hold:

$$
\begin{aligned}
& \frac{x}{3} \frac{\sqrt{1-x^{2}}+14}{2 \sqrt{1-x^{2}}+3}<\frac{1}{3} \frac{x+14(\sqrt{x+1}-\sqrt{1-x})}{3+\sqrt{x+1}+\sqrt{1-x}}<\arcsin x \\
& <\frac{(41 \sqrt{2}+25) \pi}{782} \frac{x+14(\sqrt{x+1}-\sqrt{1-x})}{3+\sqrt{x+1}+\sqrt{1-x}} \\
& <\frac{3 \pi x}{28} \frac{\sqrt{1-x^{2}}+14}{2 \sqrt{1-x^{2}}+3} \\
& \frac{3 x}{2+\sqrt{1-x^{2}}}<\frac{6(\sqrt{x+1}-\sqrt{1-x})}{4+\sqrt{x+1}+\sqrt{1-x}}<\arcsin x \\
& <\frac{(1+2 \sqrt{2}) \pi}{12} \frac{6(\sqrt{x+1}-\sqrt{1-x})}{4+\sqrt{x+1}+\sqrt{1-x}} \\
& <\frac{\pi x}{2+\sqrt{1-x^{2}}}, \\
& \frac{\pi x}{4} \frac{\sqrt{1-x^{2}}+2}{2 \sqrt{1-x^{2}}+1}<\frac{(\sqrt{2}+3) \pi}{14} \frac{x+2(\sqrt{x+1}-\sqrt{1-x})}{1+\sqrt{x+1}+\sqrt{1-x}} \\
& <\arcsin x<\frac{x+2(\sqrt{x+1}-\sqrt{1-x})}{1+\sqrt{x+1}+\sqrt{1-x}} \\
& <x \frac{\sqrt{1-x^{2}}+2}{2 \sqrt{1-x^{2}}+1} \text {. }
\end{aligned}
$$

Remark 24. Inequalities (92) are due to Zhu [23].

4.2. Inequalities for Certain Means. For $a, b>0$ with $a \neq b$, the first and second Seiffert means [24, 25]; Nueman-Sándor means [26] are defined by

$$
\begin{gathered}
P=P(a, b)=\frac{a-b}{2 \arcsin ((a-b) /(a+b))}, \\
T=T(a, b)=\frac{a-b}{2 \arctan ((a-b) /(a+b))}, \\
\text { NS }=\text { NS }(a, b)=\frac{a-b}{2 \operatorname{arcsinh}((a-b) /(a+b))},
\end{gathered}
$$

respectively. More new means can be found in [27]. We also denote the logarithmic mean, arithmetic mean, geometric mean, and quadratic mean of $a$ and $b$ by $L, A, G$, and $Q$. There has been some inequalities for these means; we quote $[7,26-$ 36]. Now we establish some new ones involving these means.

Let $x=\arcsin ((b-a) /(a+b)), \arctan ((b-a) /(a+b))$. Then $(\sin x) / x=P / A, \cos x=G / A ;(\sin x) / x=T / Q, \cos x=$ $A / Q$. And then Theorems 7, 10, 13, 14, 16 and 17 can be stated as equivalent ones involving means $P, A, G$, and $T, Q$. For example, from Theorems 7 and 17 we have the following.

Proposition 25. For $a, b>0$ with $a \neq b$, both the two-side inequalities

$$
\begin{aligned}
& \frac{2 p A+(p+3) G}{(3 p+1) A+2 G} A<P<A \frac{2 q A+(q+3) G}{(3 q+1) A+2 G}, \\
& \frac{2 p Q+(p+3) A}{(3 p+1) Q+2 A} Q<T<Q \frac{2 q Q+(q+3) A}{(3 q+1) Q+2 A}
\end{aligned}
$$

hold if and only if $p \in\left[0, p_{0}\right]$ and $q \in(-\infty,-1] \cup[9, \infty)$, where $p_{0}=(\pi-3)^{-1} \approx 7.0625$.

Making changes of variables $x=\operatorname{arctanh}((b-a) /(a+b))$, $\operatorname{arcsinh}((b-a) /(a+b))$ yield $(\sinh x) / x=L / G, \cosh x=A / G$; $(\sinh x) / x=\mathrm{NS} / A, \cosh x=\mathrm{Q} / A$, respectively. And then, Theorem 18 can be equivalently written as follows.

Proposition 26. For $a, b>0$ with $a \neq b$, both the inequalities

$$
\begin{gathered}
\frac{2 G+(1+3 p) A}{(3+p) G+2 p A} G<L, \\
\frac{2 A+(1+3 p) Q}{(3+p) A+2 p Q} A<\mathrm{NS}
\end{gathered}
$$

hold if and only if $p \in(-\infty,-1] \cup[1 / 9, \infty)$. They are reversed if and only if $p=0$.

4.3. The Estimate for the Sine Integral. For the estimations for the sine integral defined by

$$
\operatorname{Si}(x)=\int_{0}^{x} \frac{\sin t}{t} d t
$$


there has been some results (see [37-39]). By our results we can obtain many estimates for $\operatorname{Si}(x)$. Here we give a simpler but more accurate one.

Proposition 27. For $x \in(0, \pi / 2]$, we have

$$
\begin{aligned}
& \frac{4 \sqrt{2}-2}{7 \pi}\left(x+\sin x+8 \sin \frac{x}{2}\right) \\
& <\operatorname{Si}(x)<\frac{1}{6}\left(x+\sin x+8 \sin \frac{x}{2}\right) .
\end{aligned}
$$

Proof. By (63) we see that the inequalities

$$
\begin{array}{r}
\frac{4(2 \sqrt{2}-1)}{7} \frac{\cos ^{2}(t / 2)+2 \cos (t / 2)}{\pi} \\
<\frac{\sin t}{t}<\frac{\cos ^{2}(t / 2)+2 \cos (t / 2)}{3}
\end{array}
$$

hold for $t \in[0, \pi / 2]$. Integrating both sides over $[0, x]$ and simple calculation yield (98).

Remark 28. By (98) we have

$$
\begin{aligned}
1.3682 & \approx \frac{2 \sqrt{2}-1}{7 \pi}(\pi+8 \sqrt{2}+2)<\int_{0}^{\pi / 2} \frac{\sin t}{t} d t \\
& <\frac{1}{12}(\pi+8 \sqrt{2}+2) \approx 1.3713 .
\end{aligned}
$$

\section{Conflict of Interests}

The authors declare that there is no conflict of interests regarding the publication of this paper.

\section{Acknowledgments}

This research was supported by the Natural Science Foundation of China under Grants 11171307 and 61374086 and the Natural Science Foundation of Zhejiang Province under Grant LY13A010004.

\section{References}

[1] D. S. Mitrinović, Analytic Inequalities, vol. 165, Springer, New York, NY, USA, 1970.

[2] F. Qi, D.-W. Niu, and B.-N. Guo, "Refinements, generalizations, and applications of Jordan's inequality and related problems," Journal of Inequalities and Applications, vol. 2009, Article ID 271923, 52 pages, 2009.

[3] C.-P. Chen and W.-S. Cheung, "Sharp Cusa and Becker-Stark inequalities," Journal of Inequalities and Applications, vol. 2011, article 136, 6 pages, 2011.

[4] K. S. K. Iyengar, B. S. M. Rao, and T. S. Nanjundiah, "Some trigonometrical inequalities," Half-Yearly Journal of Mysore University B, vol. 6, pp. 1-12, 1945.

[5] C. Mortici, "The natural approach of Wilker-Cusa-Huygens inequalities," Mathematical Inequalities \& Applications, vol. 14, no. 3, pp. 535-541, 2011.
[6] E. Neuman and J. Sándor, "On some inequalities involving trigonometric and hyperbolic functions with emphasis on the Cusa-Huygens, Wilker, and Huygens inequalities," Mathematical Inequalities \& Applications, vol. 13, no. 4, pp. 715-723, 2010.

[7] E. Neuman, "Inequalities for the Schwab-Borchardt mean and their applications," Journal of Mathematical Inequalities, vol. 5, no. 4, pp. 601-609, 2011.

[8] J. Sándor and M. Bencze, “On Huygens' trigonometric inequality," RGMIA Research Report Collection, vol. 8, no. 3, article 14, 2005.

[9] S.-H. Wu and Á. Baricz, "Generalizations of Mitrinović, Adamović and Lazarevićs inequalities and their applications," Publicationes Mathematicae Debrecen, vol. 75, no. 3-4, pp. 447458, 2009.

[10] Z.-H. Yang, "Refinements of Mitrinović-Cusa inequality," http://arxiv.org/abs/1206.4911.

[11] Z.-H. Yang, "Renements of a two-sided inequality for trigonometric functions," Journal of Mathematical Inequalities, vol. 7, no. 4, pp. 601-615, 2013.

[12] Z.-H. Yang, "New sharp Jordan type inequalities and their applications," Gulf Journal of Mathematics, vol. 2, no. 1, pp. 110, 2014.

[13] L. Zhu, "Some new wilker-type inequalities for circular and hyperbolic functions," Abstract and Applied Analysis, vol. 2009, Article ID 485842, 9 pages, 2009.

[14] L. Zhu, "A source of inequalities for circular functions," Computers \& Mathematics with Applications, vol. 58, no. 10, pp. 19982004, 2009.

[15] C. Huygens, Oeuvres Completes 1888-1940, Sociéte Hollondaise des Science, Haga, Sweden.

[16] A. Oppenheim, "Problems for solutions E1277," The American Mathematical Monthly, vol. 64, no. 7, pp. 504-505, 1957.

[17] W. B. Carver, "Extreme parameters in an inequality," The American Mathematical Monthly, vol. 65, no. 3, pp. 206-209, 1958.

[18] Y.-F. Wu, "Remarks on generalizations of Jordan and Kober's inequalities," Studies in College Mathematics, vol. 11, no. 4, pp. 38-39, 42, 2008 (Chinese).

[19] W. D. Jiang, "On Shafer-Fink inequality and Carlson inequality," The College Mathematics Journal, vol. 23, no. 4, pp. 152-154, 2007 (Chinese).

[20] M. Li and D. He, "Better estimations for upper and lower bounds of $\sin x / x$ and its application," Journal of Beijing Union University, vol. 24, no. 2, pp. 47-48, 2010 (Chinese).

[21] R. E. Shafer, "An inequality for the inverse tangent E1867," The American Mathematical Monthly, vol. 74, no. 6, pp. 726-727, 1967.

[22] A. M. Fink, “Two inequalities," Univerzitet u Beogradu. Publikacije Elektrotehnickog Fakulteta. Serija Matematika, vol. 6, pp. 48-49, 1995.

[23] L. Zhu, "On Shafer-Fink inequalities," Mathematical Inequalities \& Applications, vol. 8, no. 4, pp. 571-574, 2005.

[24] H.-J. Seiffert, "Werte zwischen dem geometrischen und dem arithmetischen Mittel zweier Zahlen," Elemente der Mathematik, vol. 42, no. 4, pp. 105-107, 1987.

[25] H. -J. Seiffert, “Aufgabe $\beta$ 16," Die Wurzel, vol. 29, pp. 221-222, 1995.

[26] E. Neuman and J. Sándor, "On the Schwab-Borchardt mean," Mathematica Pannonica, vol. 14, no. 2, pp. 253-266, 2003. 
[27] Z.-H. Yang, "Three families of two-parameter means constructed by trigonometric functions," Journal of Inequalities and Applications, vol. 2013, article 541, 27 pages, 2013.

[28] Y.-M. Chu, Y.-F. Qiu, M.-K. Wang, and G.-D. Wang, "The optimal convex combination bounds of arithmetic and harmonic means for the Seiffert's mean," Journal of Inequalities and Applications, vol. 2010, Article ID 436457, 7 pages, 2010.

[29] Y.-M. Chu, M.-K. Wang, and Y.-F. Qiu, "An optimal double inequality between power-type Heron and Seiffert means," Journal of Inequalities and Applications, vol. 2010, Article ID 146945, 11 pages, 2010.

[30] Y.-M. Chu, M.-K. Wang, and W.-M. Gong, "Two sharp double inequalities for Seiffert mean," Journal of Inequalities and Applications, vol. 2011, article 44, 7 pages, 2011.

[31] Y.-M. Chu, B.-Y. Long, W.-M. Gong, and Y.-Q. Song, "Sharp bounds for Seiffert and Neuman-Sándor means in terms of generalized logarithmic means," Journal of Inequalities and Applications, vol. 2013, article 10, 13 pages, 2013.

[32] I. Costin and G. Toader, "A nice separation of some Seiffert-type means by power means," International Journal of Mathematics and Mathematical Sciences, vol. 2012, Article ID 430692, 6 pages, 2012.

[33] A. Witkowski, "Interpolations of Schwab-Borchardt mean," Mathematical Inequalities \& Applications, vol. 16, no. 1, pp. 193206, 2013.

[34] Z.-H. Yang, "Sharp bounds for the second Seiffert mean in terms of powermeans," http://arxiv.org/abs/1206.5494.

[35] Z.-H. Yang, "The monotonicity results and sharp inequalities for some power-type means of two arguments," http://arxiv.org/abs/1210.6478vl.

[36] Z.-H. Yang, "Estimates for Neuman-Sándor mean by power means and their relative errors," Journal of Mathematical Inequalities, vol. 7, no. 4, pp. 711-726, 2013.

[37] F. Qi, “Extensions and sharpenings of Jordan's and Kober's inequality," Journal of Mathematics for Technology, vol. 12, no. 4, pp. 98-102, 1996 (Chinese).

[38] S. Wu and L. Debnath, "A new generalized and sharp version of Jordan's inequality and its applications to the improvement of the Yang Le inequality," Applied Mathematics Letters, vol. 19, no. 12, pp. 1378-1384, 2006.

[39] S.-H. Wu, "Sharpness and generalization of Jordan's inequality and its application," Taiwanese Journal of Mathematics, vol. 12, no. 2, pp. 325-336, 2008. 


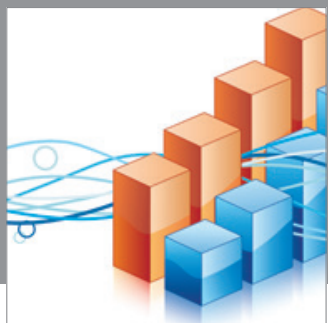

Advances in

Operations Research

mansans

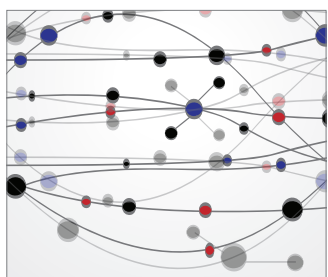

The Scientific World Journal
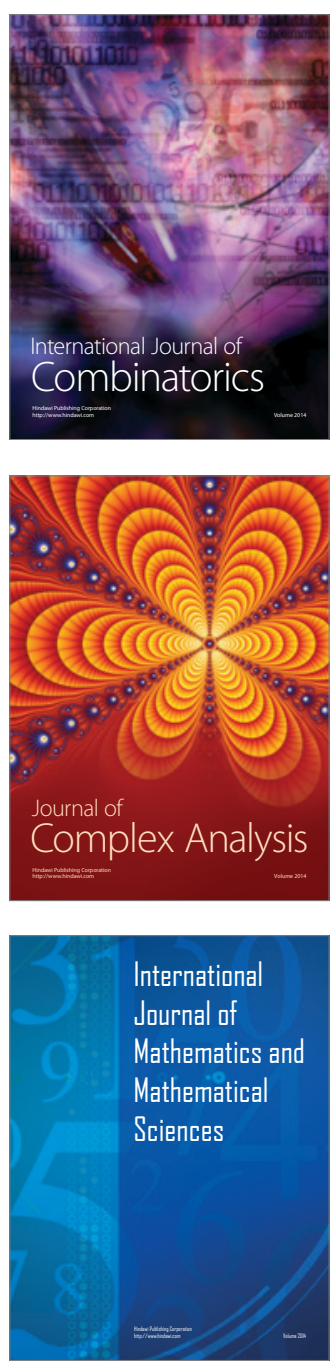
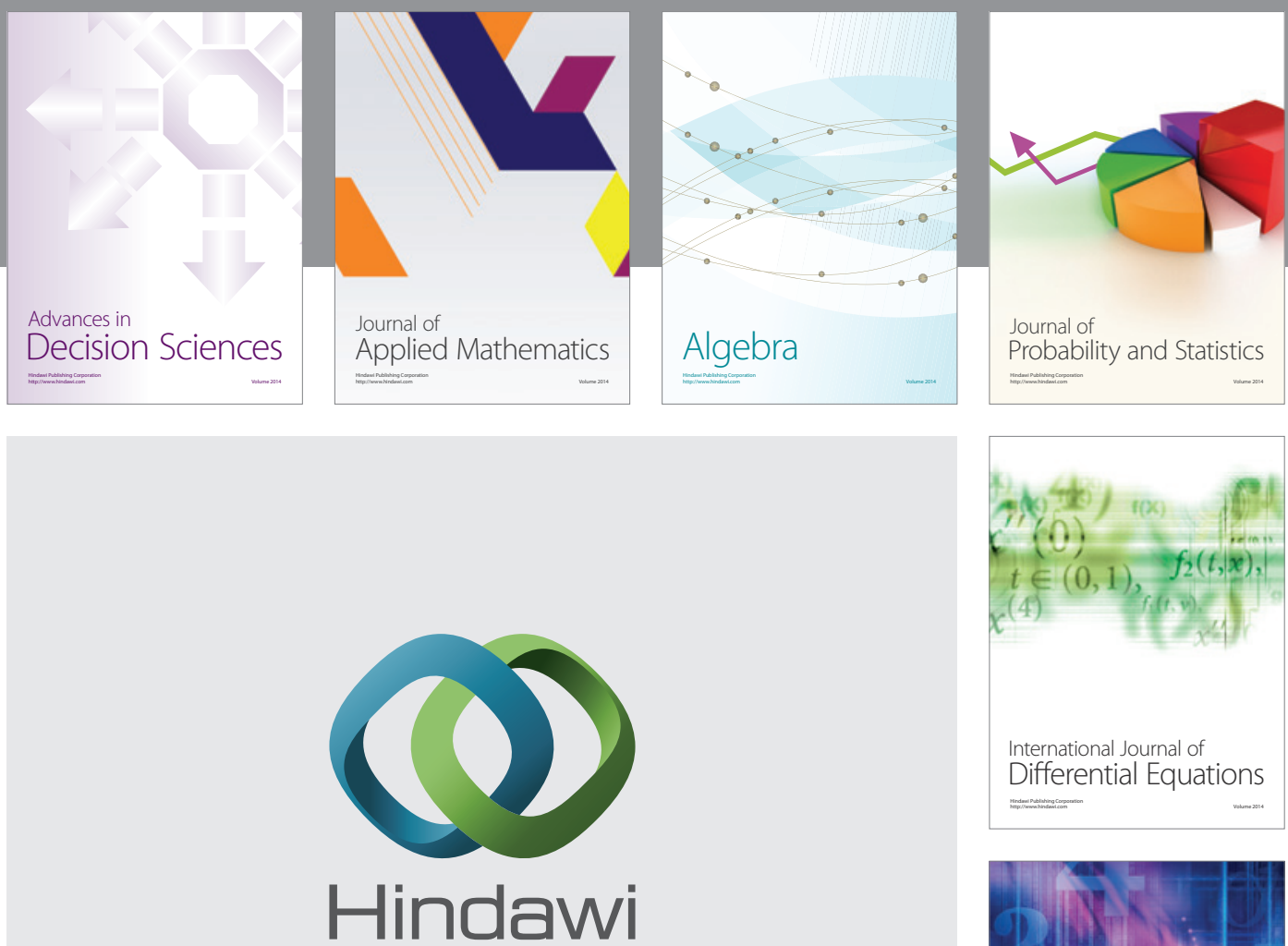

Submit your manuscripts at http://www.hindawi.com
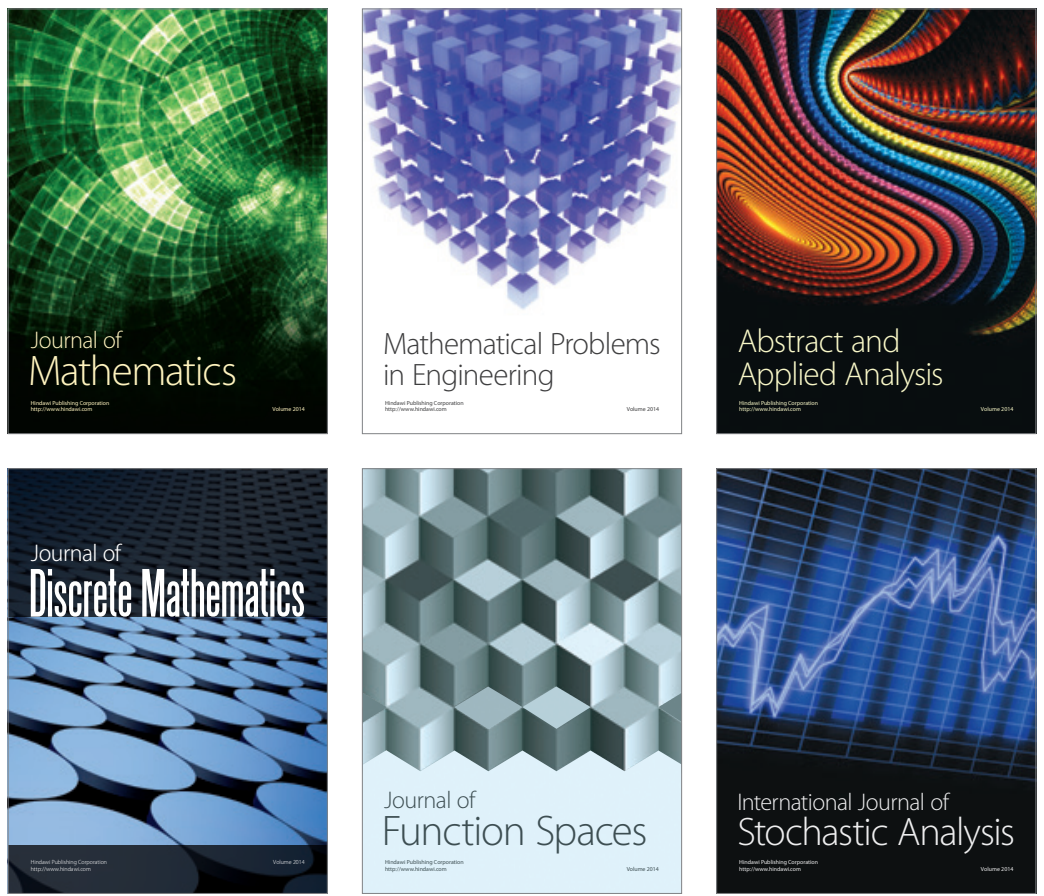

Journal of

Function Spaces

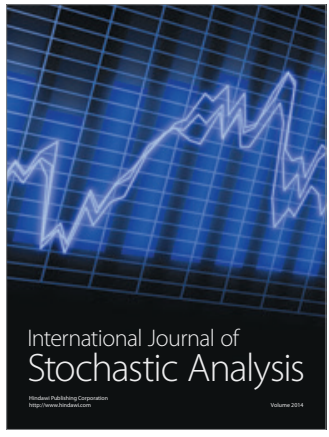

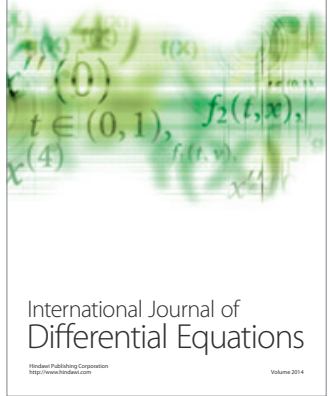
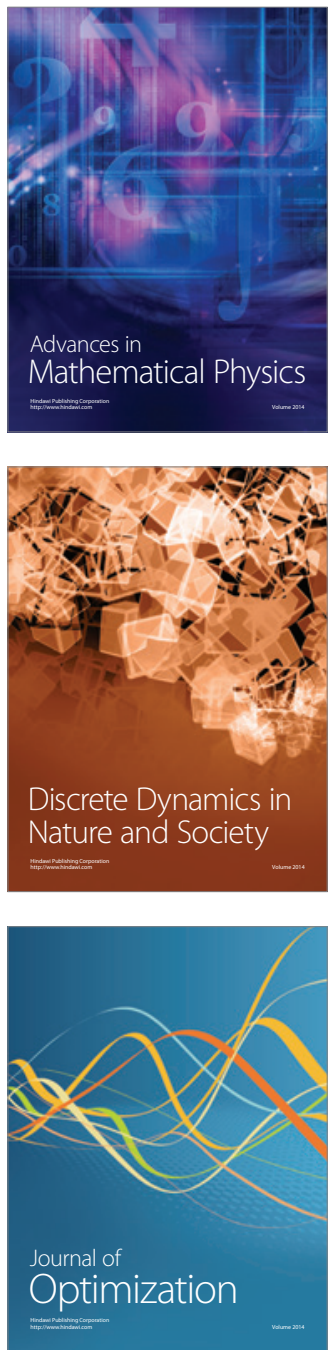